\title{
CFD modelling of liquid-liquid multiphase microstructured reactor: Slug flow generation
}

\author{
M.N. Kashid*, A. Renken, L. Kiwi-Minsker \\ Group of Catalytic Reaction Engineering (GGRC), Ecole Polytechnique Fédérale de Lausanne (EPFL), Station 6, CH - 1015 Lausanne, \\ Switzerland
}

\begin{abstract}
A B S T R A C T
Microreactor technology, an important method of process intensification, offers numerous potential benefits for the process industries. Fluid-fluid reactions with mass transfer limitations have already been advantageously carried out in small-scale geometries. In liquid-liquid microstructured reactors (MSR), alternating uniform slugs of the twophase reaction mixture exhibit well-defined interfacial mass transfer areas and flow patterns. The improved control of highly exothermic and hazardous reactions is also of technical relevance for large-scale production reactors. Two basic mass transfer mechanisms arise: convection within the individual liquid slugs and diffusion between adjacent slugs. The slug size in liquid-liquid MSR defines the interfacial area available for mass transfer and thus the performance of the reactor. There are two possibilities in a slug flow MSR depending on the interaction of the liquids with the solid wall material: a dispersed phase flow in the form of an enclosed slug in the continuous phase (with film—complete wetting of the continuous phase) and an alternate flow of two liquids (without film—partial wetting of the continuous phase). In the present work, a computational fluid dynamics (CFD) methodology is developed to simulate the slug flow in the MSR for both types of flow systems. The results were validated with the experimental results of Tice et al. (J.D. Tice, A.D. Lyon and R.F. Ismagilov, Effects of viscosity on droplet formation and mixing in microfluidic channels, Analytica Chimica Acta 507 (1) (2004), pp. 73-77.).
\end{abstract}

( 2010 The Institution of Chemical Engineers. Published by Elsevier B.V. All rights reserved.

Keywords: Computational fluid dynamics; Volume of fluid; Microstructured reactor; Slug flow

\section{Introduction}

The liquid-liquid slug flow capillary microstructured reactor (MSR) has been demonstrated to be a useful instrument for the enhancement of heat and mass transfer limited reactions (Dummann et al., 2003). The internal circulations within both the slugs caused by the shear between the wall surface and the slug axis renew the interface and enhance the diffusive penetration, and consequently, increase the observed reaction rates. The frequency of surface renewal varies with slug size-the lower the slug size, the higher the frequency. The slug size in a MSR depends on the two fluid mixing geometry and the physical properties of fluids. The small slugs are in the range of microliters giving very high interfacial area for mass transfer as compared to its conventional counterpart. Thus, for the rational design of such a reactor, a precise investigation of slug size and interfacial area is required to achieve the optimal performance of liquid-liquid slug flow MSR.

Several MSR with different types of two-phase mixing elements such as T-type (Burns and Ramshaw, 1999; Zhao et al., 2007) and Y-type (Dummann et al., 2003; Kashid, 2007) have been used for experimentation in the laboratory. Garstecki et al. $(2006 \mathrm{a}, \mathrm{b})$ used a T-type mixing element where the dispersed phase was introduced in the continuous flow channel at a right angle and proposed a correlation for slug length as a function of the ratio of dispersed to continuous phase flow (flow rate range: $10^{-2}$ to $1 \mu \mathrm{L} / \mathrm{s}$ in a microchannel of diameter of $100 \mu \mathrm{m}$ which corresponds to the flow velocity of $1-100 \mu \mathrm{m} / \mathrm{s}$ ). The interfacial forces are highly dominant and the correlation was found to be independent of flow velocity. However, the experimental studies of Tice et al. (2004) clearly show the effect of flow velocity on the slug size. Recently, Dessimoz et al. (2008) used both types of mixing elements ( $\mathrm{T}$ and $\mathrm{Y}$ ) and

\footnotetext{
* Corresponding author.

E-mail address: madhvanand.kashid@epfl.ch (M.N. Kashid).

Received 16 January 2009; Received in revised form 25 November 2009; Accepted 29 November 2009 0263-8762/\$ - see front matter (c) 2010 The Institution of Chemical Engineers. Published by Elsevier B.V. All rights reserved. doi:10.1016/j.cherd.2009.11.017
} 
reported the effect of mixing element on the two-phase flow patterns. The pressure drop across the mixing element has a significant contribution in the overall energy requirement. In the case of Y-type element the pressure drop is less when compared with T-type mixing elements as frictional losses are lower in the former case. Thus, to understand the slug flow generation mechanism and the effect of different operating conditions on the slug size, more work is needed.

The CFD based models can be coupled with phenomenological models and experimental observations to develop a complete toolkit of models, which can give a clear picture of the fluid dynamics, heat and mass transfer in MSR thereby helping to improve the performance of the reactors. The use of CFD to study hydrodynamics and mass transfer in fluid-fluid MSR has been exploited only to a limited extent. The previous numerical investigation includes the volume of fluid (VOF) methodology to demonstrate the internal circulations and slug flow (Kashid et al., 2007a) and a unit cell based model for mass transfer with and without chemical reaction (Kashid et al., 2007b). The latter study was restricted to a unit cell due to lack of computational power. Further, Qian and Lawal (2006) and Kumar et al. (2007) investigated the hydrodynamics of the Taylor flow in gas-liquid two-phase flow using VOF method. All above publications neglect the liquid film on the capillary wall which is not the real picture in many cases. Recently, Gupta et al. (2009) developed a numerical methodology to model gas-liquid Taylor flow in microchannels for a core-annular feed inlet and discussed numerical details for the modelling of wall film. The results obtained were compared with experimental correlations in the literature and good agreement was found. Thus, the literature shows a very few studies addressing the underlying physics of the two-phase slug flow generation which is not enough for the rational design of a MSR.

In the present work, a VOF based CFD methodology is developed to investigate the slug flow generation in a T-type mixing element considering the wall film. The computational methodology along with the underlying physics is explained in detail. Finally, the results obtained are compared with the experimental results of Tice et al. (2004).

\section{Numerical model}

\subsection{Governing equations}

Different methods are available to model immiscible fluids such as level set, volume of fluid, marker particle, lattice Boltzmann, front tracking and so on (Van Sint Annaland et al., 2006). VOF and level set methods belong to the most common implicit free surface reconstruction methods, while particularly VOF is relatively simple to treat topological changes of the interface and is naturally conservative. This method was extensively used for many applications (e.g., Bothe et al., 2003; Cerne et al., 2001; Chen and Li, 1998; Li and Renardy, 2000). The VOF methodology developed by Hirt and Nichols (1981) relies on the fact that two or more phases are not interpenetrating and for each additional phase the volume fraction of the phase is added in the computational cell. In each control volume, the volume fractions of all the phases sum up to unity. This methodology has several advantages: reasonable accuracy, relative simplicity and ability to solve complex free surface flows.
The VOF methodology described in commercial CFD package FLUENT (ANSYS Inc.) was used (Fluent, 2006). For the isothermal flow of two Newtonian fluids of constant density which are immiscible on the molecular scale, the governing equations for velocity $(u)$ and pressure $(p)$ can be written as follows (incompressible Navier-Stokes equations):

$$
\begin{aligned}
& \frac{\partial \mathbf{u}}{\partial \mathrm{t}}+\nabla \cdot(\mathbf{u u})=-\frac{1}{\rho}\left(\nabla p+\mu \nabla^{2} \mathbf{u}\right)+\frac{1}{\rho} \mathbf{F}_{\mathrm{SF}} \\
& \nabla \cdot \mathbf{u}=0
\end{aligned}
$$

where $F_{\mathrm{SF}}$ is the continuum surface force (CSF) vector. The above equation is dependent on the volume fractions of all the phases through the properties $\rho$ and $\mu$. The interface between two fluids was tracked by the volume fraction, $\alpha_{k}$. The evolution of this function can be formulated as a general transport problem. The conservation of this function can be represented with the help of interface mass balance conditions using a convection equation:

$\frac{\partial \alpha_{k}}{\partial \mathrm{t}}+\mathbf{u} \cdot \nabla \alpha_{k}=0$

The above equation is usually solved for the secondary phase which is defined based on the experimental observations. In the liquid-liquid slug flow system, the fluid flowing in the form of an enclosed slug is considered as the secondary phase. Without experimental evidence, it can also be judged from the wetting properties (hydrophilic/hydrophobic) of the channel wall.

In a conventional tubular reactor (diameter $\geq 3 \mathrm{~mm}$ ), gravity shows a considerable effect on flow patterns while the surface tension effects are quite low. But in MSR, the flow patterns are influenced mainly by surface tension, viscous and inertial effects while the gravitational effects are quite low. Therefore, modelling of surface tension and wall adhesion effects are important in addition to mass and momentum transfer. In the present work, the CSF model proposed by Brackbill et al. (1992) was used to model surface tension. The surface curvature was computed from the local gradients in the surface normal at the interface. Thus, the source term in the momentum equation was specified as follows:

$\mathbf{F}_{\mathrm{SF}}=\sigma \kappa \mathbf{n}\left[\frac{\alpha_{1} \rho_{1}+\alpha_{2} \rho_{2}}{(1 / 2) \rho_{1}+\rho_{2}}\right]$

where $\mathbf{n}$ is the surface normal and $\kappa$ is the curvature which are given as

$$
\begin{aligned}
& \mathbf{n}=\nabla \alpha_{2} \\
& \kappa=-(\nabla \cdot \hat{\mathbf{n}})=\frac{1}{|\mathbf{n}|}\left[\left(\frac{\mathbf{n}}{|\mathbf{n}|} \cdot \nabla\right)|\mathbf{n}|-(\nabla \cdot \mathbf{n})\right]
\end{aligned}
$$

The surface normal $\mathrm{n}$ was evaluated in the interface containing cell. The geometric reconstruction scheme (piecewise linear interface calculation, PLIC) was used to calculate the interface position in the cell. When compared with other schemes, it gives relatively low numerical diffusion and therefore it is widely used. Simulations were carried out for two types of flow cases: complete and partial wetting of continuous fluid to the wall. If $\theta_{\mathrm{dcw}}$ is the contact angle, then the surface normal at the cell next to the wall is

$\hat{\mathbf{n}}=\hat{\mathbf{n}}_{w} \cos \theta_{d c w}+\hat{\mathbf{t}}_{w} \sin \theta_{d c w}$ 


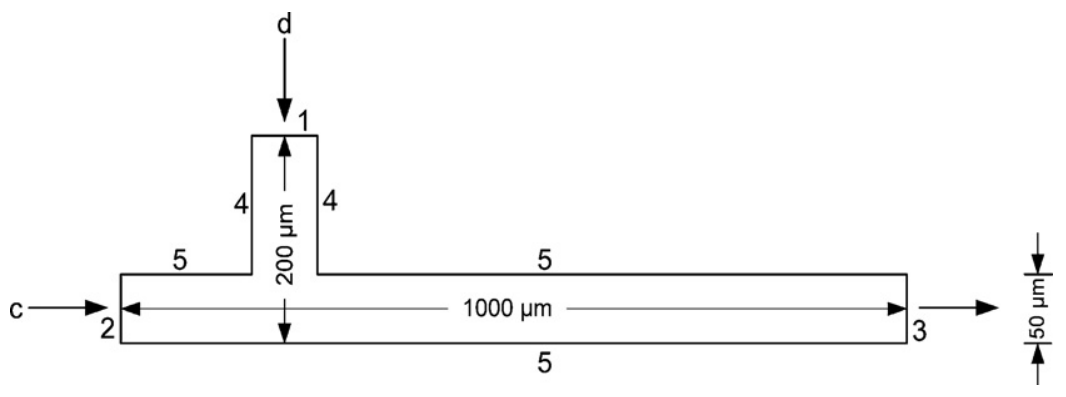

Fig. 1 - Schematic of the geometry and the boundary conditions used in the present work (c: continuous phase; d: dispersed phase; 1: velocity inlet condition for dispersed phase; 2: velocity inlet condition for continuous phase; 3: pressure outlet condition; 4: complete wetting wall boundary for dispersed phase $\left(\theta_{\mathrm{dcw}}=0^{\circ}\right)$; 5: complete $\left(\theta_{\mathrm{dcw}}=180^{\circ}\right) /$ partial $\left(\theta_{\mathrm{dcw}} \neq 0^{\circ}\right.$ or $180^{\circ}$ ) wetting wall condition for continuous phase).

\subsection{Numerical mesh, boundary conditions and solution}

A T-type geometry and boundary conditions used are shown in Fig. 1. The mesh was generated using the pre-processor GAMBIT software (ANSYS Inc.). A structured mesh was generated and the planer (2D) geometry considered due to very fine mesh size required for resolving the wall film. Different mesh sizes were used in order to make the solution mesh independent. The physical properties and simulation parameters used in the present work are listed in Table 1. A uniform inlet velocity was set at the two inlets and atmospheric pressure was set at the outlet. A no-slip boundary condition was used at the walls where the velocity is zero.

The PISO (pressure-implicit with splitting of operators) was used for pressure velocity coupling which reduces the internal iteration per time step and allows larger under relaxation parameters. The PRESTO! (pressure staggering option) for the pressure interpolation and second order upwind differencing were used for solving transport equations. The geometric reconstruction scheme was used for the interface reconstruction and implicit body force treatment for the body force formulation. The absolute convergence criterion for the continuity equation and the velocity was 0.001 . The simulations were carried out on a desktop computer (Intel core $2 \mathrm{CPU}$, $2.39 \mathrm{GHz}$ and $3 \mathrm{~GB}$ RAM). The typical simulation time for a mesh with 58,000 cells was about 4 days. The maximum

Table 1 - Physical properties of liquids used for the simulations.

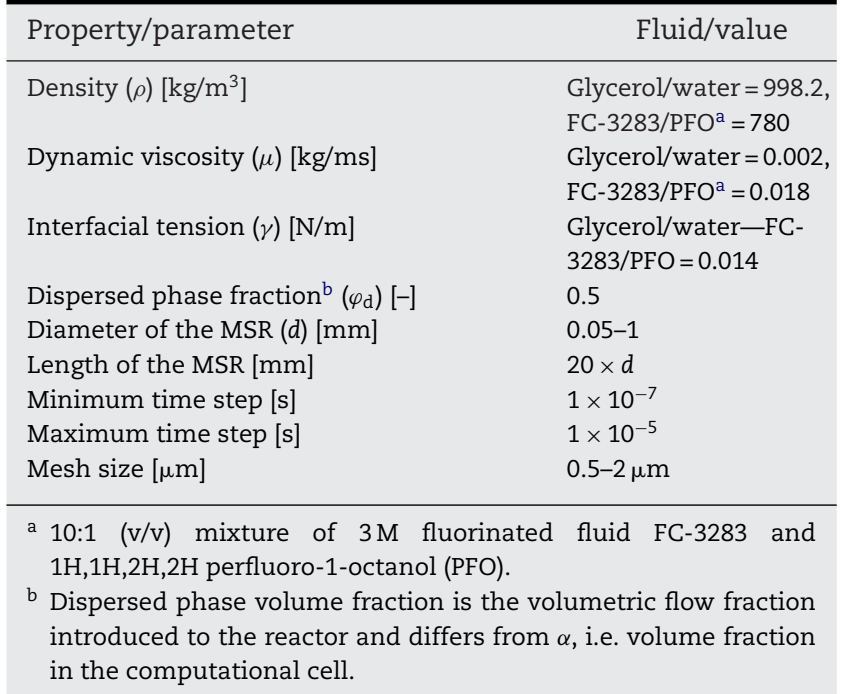

Courant number $\left(C_{r}\right)$ of 0.25 was used for the volume fraction calculation which is defined as

$$
C_{r}=U \frac{\Delta t}{\Delta l}
$$

where $U, \Delta t$ and $\Delta l$ are the average flow velocity (total flow rate/cross-sectional area), the maximum time step size and the mesh size, respectively.

\section{Results and discussion}

Two liquids were introduced into the mixing element considering that the MSR is initially filled with the continuous phase. In the laboratory experiments, two immiscible liquids are introduced into the reactor either at the same time or initially continuous phase flows for some time and then the dispersed phase is introduced. The interpenetration of two streams in the vicinity of the junction generates the characteristic alternating slug flow structure. For a given set of operating conditions, different simulations were carried out by varying the mesh size. It was observed that the mesh independent solutions were obtained for mesh sizes $(\Delta l)$ from $0.5 \mu \mathrm{m}$ to $2 \mu \mathrm{m}$ depending on the operating conditions.

\subsection{Steps of slug flow generation}

When the dispersed phase enters the main channel of continuous phase, a well-defined slug flow is achieved in five stages (Fig. 2) as explained as follows:

(a) Initially the dispersed phase penetrates the main stream and the drop begins to grow.

(b) As the tip of the dispersed phase grows, it blocks almost all the cross-sectional area of the main channel which in turn builds pressure upstream. The radius of the tip curvature is limited by the dimensions of the channel. The continuous phase flows in the gap between the wall and the dispersed phase drop with higher flow velocity.

(c) Due to the shear exerted by the continuous fluid on the droplet and the pressure drop along the length of the channel, the drop elongates and grows downstream. The interface approaches the downstream inlet of the dispersed phase and a slug (droplet with a length greater than the channel diameter) is formed in the main channel.

(d) The neck of the immiscible liquid gets squeezed further, i.e. the radial curvature dominates the axial and the slug separates from the dispersed phase stream. 


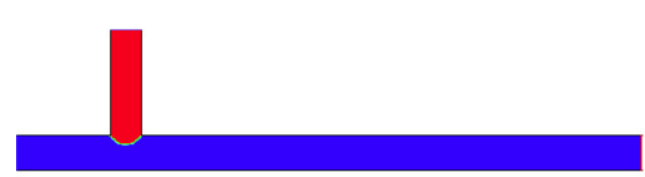

(a) $\mathrm{t}=11.7 \mathrm{~ms}$

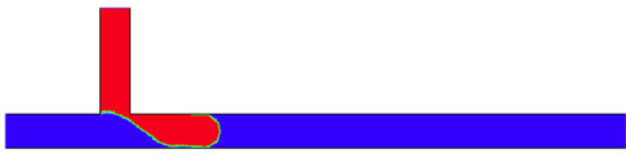

(c) $\mathrm{t}=19.1 \mathrm{~ms}$

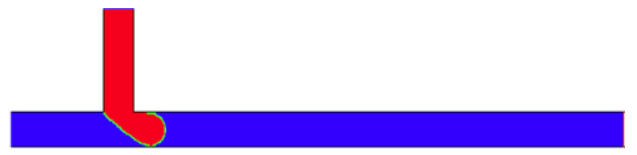

(b) $\mathrm{t}=15.6 \mathrm{~ms}$

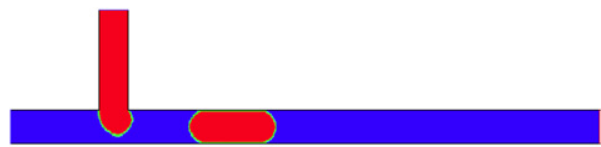

(d) $\mathrm{t}=22.9 \mathrm{~ms}$

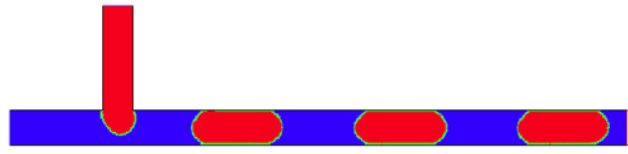

(e) $\mathrm{t}=41.4 \mathrm{~ms}$

Fig. 2 - Five steps of slug flow generation $(U=27 \mathrm{~mm} / \mathrm{s}, \gamma=0.014 \mathrm{~N} / \mathrm{m}$, and $d=50 \mu \mathrm{m})$.

The processes of drop penetration and separation from the dispersed phase continue and produce a well-defined slug flow.

The pressure behaviour in the continuous phase inlet and the dispersed phase inlet is shown in Fig. 3. Three forces act on the tip of the discontinuous phase, the force arising from the increased resistance to flow of the discontinuous fluid around the tip, the interfacial tension force, and the shear stress force of the continuous phase (Garstecki et al., 2006a), which are explained in the following section.
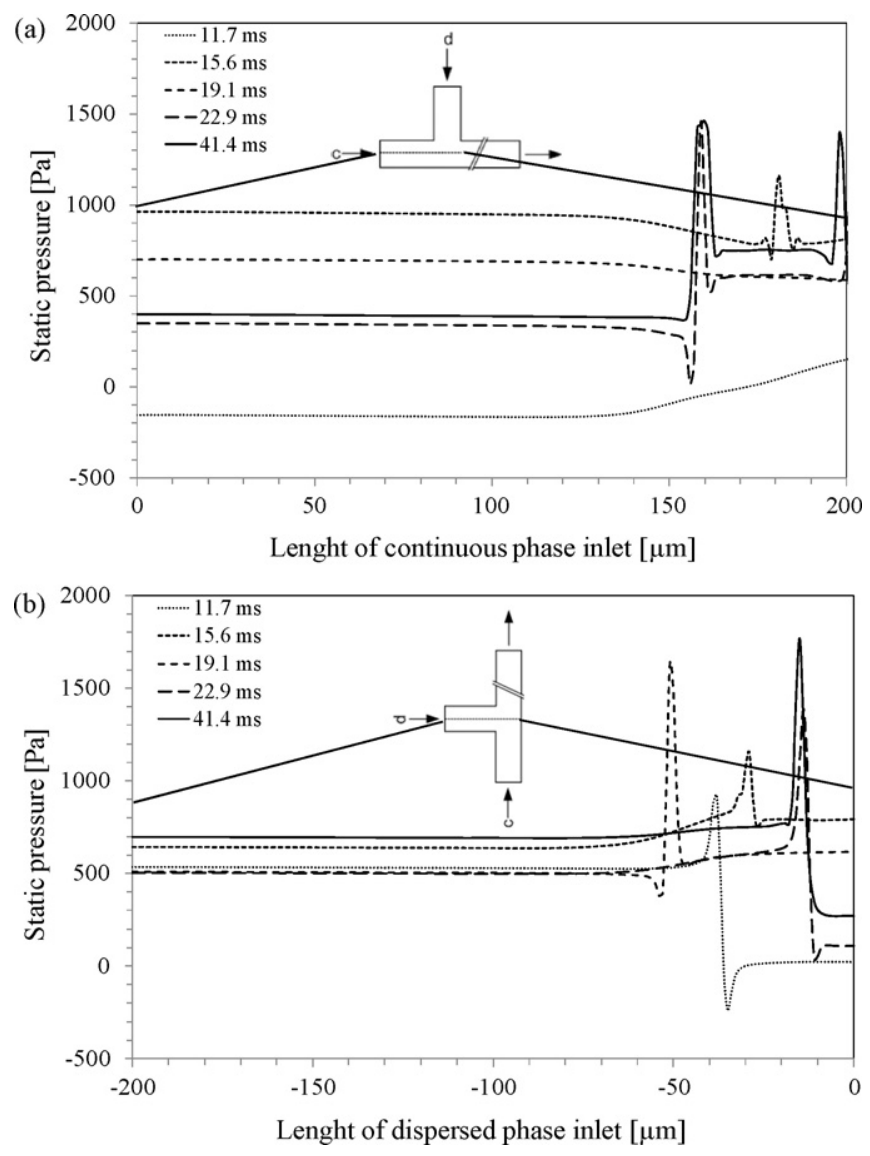

Fig. 3 - Behaviour of static pressure for the five steps in Fig. 2. (a) Along the length of continuous phase channel and (b) Along the length of dispersed phase inlet $(U=27 \mathrm{~mm} / \mathrm{s}, \gamma=0.014 \mathrm{~N} / \mathrm{m}$, and $d=50 \mu \mathrm{m})$.
In the first stage of the drop penetration, the pressure in the continuous phase inlet is lower in the upstream part and increases in the vicinity of the junction due to the decreased flow cross-section. When the pressure in continuous and dispersed phase are compared in the vicinity of the T-junction, the former (Fig. 3a) is relatively lower compared to the latter (Fig. 3b). When the drop occupies the entire cross-section of the channel (stage b), high pressure is built up in the upstream region and when the droplet elongates and grows downstream, the pressure again reduces (stage c). These pressure fluctuations in the continuous phase achieve an average value in the case of well-defined slug flow. However, the pressure in the dispersed phase inlet remains almost constant during all stages of the slug flow formation (Fig. 3b).

The Laplace pressure computed from the average pressure drop across the front interface and the back interface is plotted in Fig. 4. The points were randomly selected across the interface in the marked area and the average value was investigated considering pressure drops at three positions: dispersed phase inlet, across the whole emerging droplet and across the separated slugs. Though these calculations underestimate the Laplace pressure due to 2D simulations, but it is useful for qualitative analysis of slug flow generation mechanism. The pressure drop at the dispersed phase inlet clearly shows the slug flow generation mechanism. As the drop penetrates in the main stream, the Laplace pressure decreases as the curvature shape changes from the outer direction to the inner because the pressure in the continuous phase increases. In the third stage, pressure in the continuous phase becomes higher than in the dispersed phase, the Laplace pressure shows a negative value and the curvature completely changes the shape. As the neck of the immiscible thread breaks, the tip of the dispersed phase recoils to the inlet channel and the pressure in the continuous phase drops and therefore the Laplace pressure again comes back to its initial value.

When the Laplace pressure is computed across the emerging droplet, it shows a different trend with increased pressure in step (b) and subsequently decreasing behaviour in steps (c) and (d). The Laplace pressure for dispersed phase inlet and across emerging droplet is identical in stepes (a) and (d) because in both cases the droplet under investigation is penetrating in the continuous flow stream, i.e. the pressure points are similar. In the case of well-defined slug flow, the average pressure drop across the first droplet separated in the fourth step (d) and a slug of the well-defined slug flow was investigated (step e). Each slug gives a pressure drop of more than 

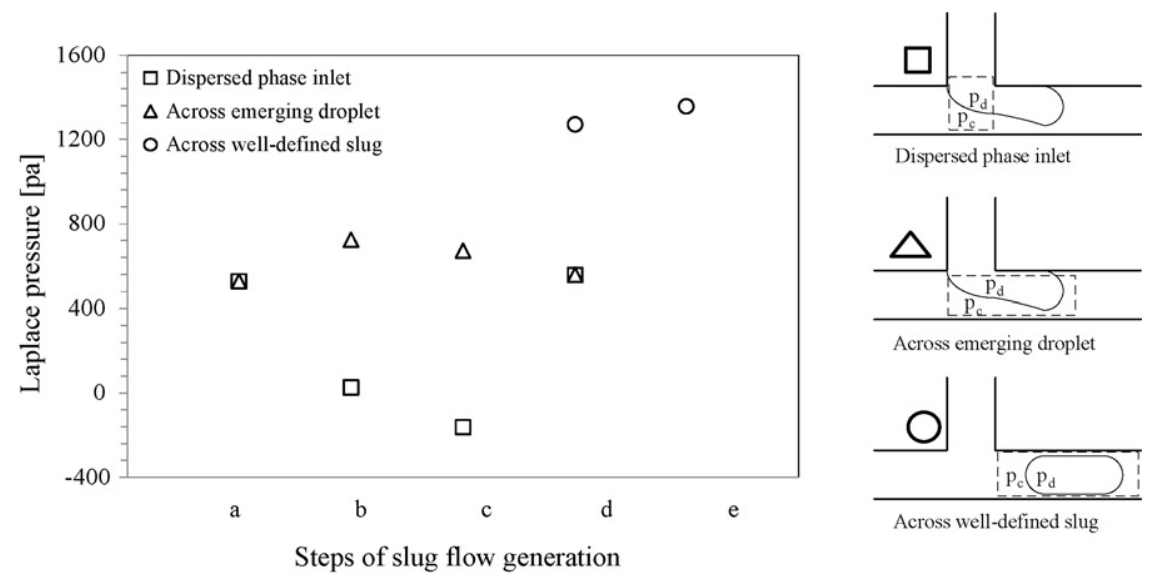

Fig. 4 - Laplace pressure in different steps of slug flow generation $(U=27 \mathrm{~mm} / \mathrm{s}, \gamma=0.014 \mathrm{~N} / \mathrm{m}$, channel diameter $=50 \mu \mathrm{m})$. The Laplace pressure was calculated over the marked domain as the difference between the pressure in the continuous $\left(p_{c}\right)$ and in the dispersed $\left(p_{d}\right)$ phase.

$1200 \mathrm{~Pa}$ in the well-defined slug flow (Fig. 4). This information is useful to investigate the total pressure drop in the slug flow channel if the slug length is known.

A clearer picture of the pressure behaviour in a well-defined slug flow is given in Fig. 5. The dispersed phase shows higher pressure while the continuous phase shows lower pressure and the difference between the two values is the Laplace pressure. Thus, the overall pressure in the liquid-liquid slug flow has two contributions: pressure drop due to viscous contribution and pressure drop due to interfacial tension (Laplace pressure). The surface force which acts against the pressure force and shear force of continuous fluid can be estimated from the height of the channel. Since the curvature of the emerging drop is bounded by the height of the channel, the total surface tension force can be calculated by multiplying the surface tension coefficient by the height of the channel.

The shear force of the continuous fluid on the tip of the dispersed phase plays an important role in drop deformation. The shear stress $(\tau)$ and shear force $\left(F_{\tau}\right)$ can be estimated by the following equations (Garstecki et al., 2006a):

$$
\begin{aligned}
& \tau=\mu \frac{u_{\text {film }, i}}{h_{i}} \\
& F_{\tau}=\mu \frac{u_{\text {film }, i} L_{d, i} d}{h_{i}}
\end{aligned}
$$

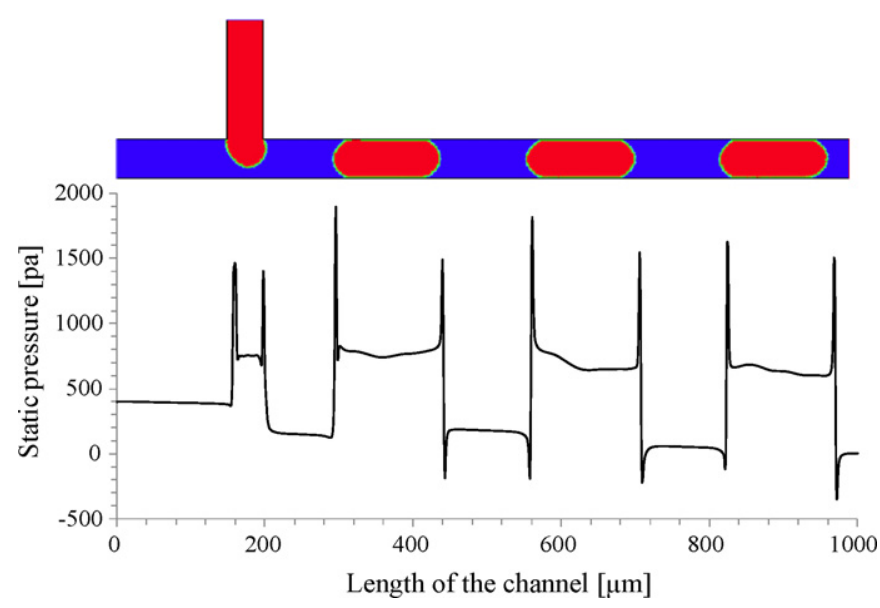

Fig. 5 - Behaviour static pressure along the length of the channel in well-defined slug flow system $(U=27 \mathrm{~mm} / \mathrm{s}$, $\gamma=0.014 \mathrm{~N} / \mathrm{m}$, and $d=50 \mu \mathrm{m})$. where $u_{\text {film }}, h$ and $L_{d}$ are film velocity, film thickness and length of dispersed slug. The subscript $i$ denotes that these values are during the slug flow generation (stages $b$ and c). The film thickness in this case is the gap between the emerging droplet of the dispersed phase and the wall. Considering the film thickness obtained from the CFD simulations, the values of shear force in steps $b$ and $c$ are about $6 \times 10^{-7} \mathrm{~N}$ and $1.26 \times 10^{-6} \mathrm{~N}$, respectively. This force along with the force of the continuous phase (calculated from CFD simulated pressure values, step $b-1.78 \times 10^{-7} \mathrm{~N}$ and step $c-1.45 \times 10^{-6} \mathrm{~N}$ ) acts against the surface tension force (minimum value for steps $b$ and $c-7 \times 10^{-7} \mathrm{~N}$ ). Thus, it shows that if the pressure exerted by the continuous phase is about one order of magnitude higher than the surface tension force, the slug is generated in the vicinity of the junction.

\subsection{Wetting behaviour}

Wetting in liquid-liquid slug flow is still an unresolved issue. If the surface tension between one of the liquid phases and the wall material is higher than the interfacial tension between the two liquids, that liquid flows in the form of an enclosed slug, while the other flow in the form of a continuous phase forming a thin wall film of a few micrometers in size. If the flow does not satisfy these conditions then both liquids flow alternatively-wall is wetted by both the fluids. In both the cases, the surface tension at the liquid-liquid interface must be sufficiently high in order to avoid destruction of slugs by the shear (Tice et al., 2003). Though partial wetting phenomenon is not widely accepted, in the chemical analysis of wall film (Kashid, 2007) it was observed that liquid-liquid slug flow does not show the film in all parts of the channel or the film is not active during the chemical reaction. In order to understand this effect of partial wetting on slug flow generation, simulations were carried out for two cases: complete wetting and partial wetting of the channel by the continuous fluid.

The comparison of slug flow for complete and partial wetting of the continuous phase for the same flow velocity and interfacial tension is shown in Fig. $6 a$ and b. In the case of partial wetting, the dispersed phase sticks to the wall for a longer time and produces bigger slugs as compared to the case of complete wetting of the continuous phase. In our simulations, the slug length observed for partial wetting $\left(\theta_{\mathrm{dcw}}=120^{\circ}\right)$ was about $20 \%$ higher than the case of complete wetting. Though the contact angle was defined, the simulations for partial wet- 
(a)

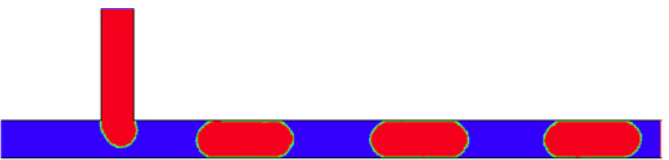

(b)

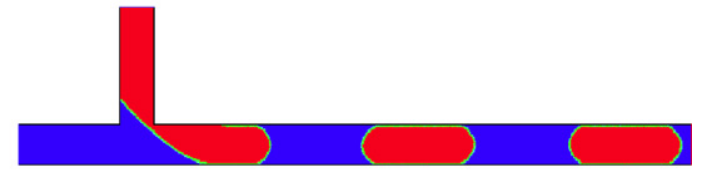

(c)

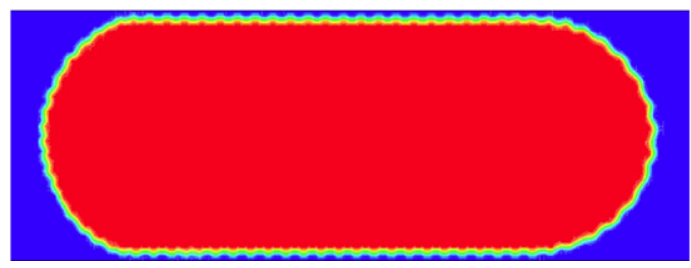

Fig. 6 - (a) Simulated snapshot of well-defined slug flow using complete wetting by continuous fluid condition, (b) simulated snapshot of well-defined slug flow using three phase contact angle (partial wetting) of $120^{\circ}$ and (c) magnified image of a single slug showing dispersed phase surrounded by a wall film in both cases of complete and partial wetting $(U=27 \mathrm{~mm} / \mathrm{s}, \gamma=0.014 \mathrm{~N} / \mathrm{m}$, and $d=50 \mu \mathrm{m})$.

ting also show a wall film. The magnified view of the simulated snapshot shows a film between the enclosed slug and the wall (Fig. 6c). The film behaviour was similar for both the cases of complete and partial wetting. In the present simulations, the film thickness obtained was about $2 \mu \mathrm{m}$ and is in the range of Bretherton's law (Bico and Quere, 2000). It also shows the interface thickness of almost one cell size because of numerical diffusion which can be reduced with further mesh refinement.

\subsection{Experimental validation}

The microscopic observations for slug flow generation reveal that the phenomenon can be characterised by the Laplace pressure, pressure of the continuous fluid and shear stress of the continuous fluid on the dispersed phase. However, in the experimental work it is difficult to characterise these forces precisely. Therefore a broader picture of slug flow generation is explained in this section from the experimental results of Tice et al. (2004). They proposed a mechanism for the slug flow formation based on the Capillary number (Ca) which is defined as

$\mathrm{Ca}=\frac{\mathrm{U} \mu}{\gamma}$

The investigation was done in a T-type reactor where the continuous phase (less viscous, dynamic viscosity of $2.0 \mathrm{mPa} \mathrm{s}$ ) flows in the straight channel while the dispersed phase (more viscous, dynamic viscosity of $18 \mathrm{mPa}$ ) meets at a right angle. The Reynolds number was low $(\mathrm{Re}<1$, defined based on the properties of continuous fluid) and these Re values have a negligible effect on the formation of slugs and mixing inside them (Tice et al., 2003).

The experimental snapshots of the slug flow formation adapted from Tice et al. (2004) are depicted in Fig. 7. It shows different slug break-up lengths which they refer to as flow regimes namely slug flow, threshold and laminar flow. In the first regime, slugs are immediately separated from the aqueous stream at the junction where the aqueous stream meets (a)

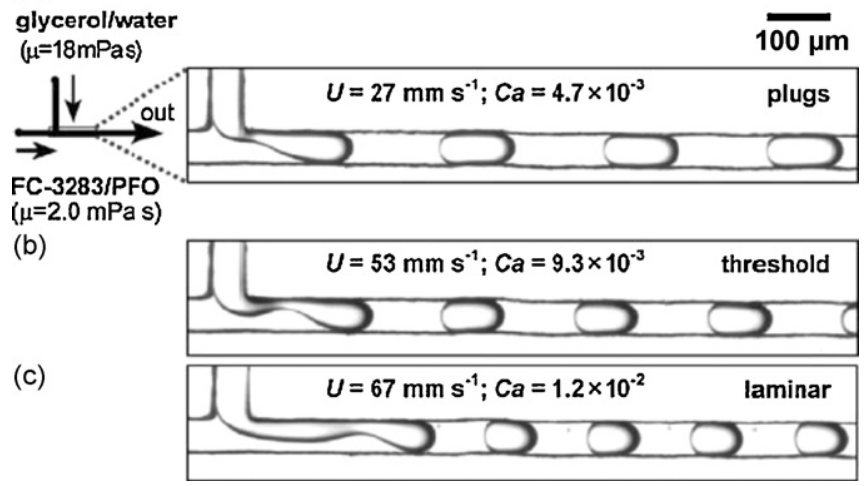

Fig. 7 - Visualization of the three regimes of behaviour observed in two-phase flows of viscous fluids (glycerol/water-FC-3283/PFO). (a) Ca $=4.7 \times 10^{-3}$. (b) $\mathrm{Ca}=9.3 \times 10^{-3}$. (c) $\mathrm{Ca}=1.2 \times 10^{-2}\left(\varphi_{\mathrm{d}}=0.5\right.$, $d=50 \mu \mathrm{m} \times 50 \mu \mathrm{m})$. Adapted from Tice et al. (2004) (Reproduced with permission. Copyright Elsevier, 2004).

the stream of the carrier fluid. The flow was very well defined and reproducible. As the flow velocity and Ca were increased to a certain threshold, slugs did not separate immediately at the junction. Instead, the aqueous stream formed a short (one to two times the width of the channel) laminar segment beyond the junction before slugs separated from the stream. In the last case of higher flow velocities and $\mathrm{Ca}$, the laminar segment propagated further downstream and its length fluctuated.

The simulated snapshots corresponding to the experimental operating conditions of Tice et al. (2004) are depicted in Fig. 8. The results show very good agreement at low flow velocity where the clear slug formation takes place in the vicinity of the T-junction. However, the performance of the CFD simulations deviates from the experimental values at high flow velocities. In the case of $67 \mathrm{~mm} / \mathrm{s}$, the slug length remained the same as that of $53 \mathrm{~mm} / \mathrm{s}$ which is very high as compared to the experimental results. This is because of the limitations of mesh size for the computations. It is believed that with a further decrease in the mesh by at least a factor of 2 will be able to capture this behaviour. Thus, the CFD results show only very good agreement with experiments at low flow velocity.

Tice et al. (2004) explained these flow regimes in terms of interfacial velocity $(\gamma / \mu)$. If the flow velocity is much lower than the interfacial velocity, the clean slug formation takes place and as the velocity of the flow increases, the interfacial velocity is not high enough, and fluids proceed laminar for some distance before the snap-off occurs.

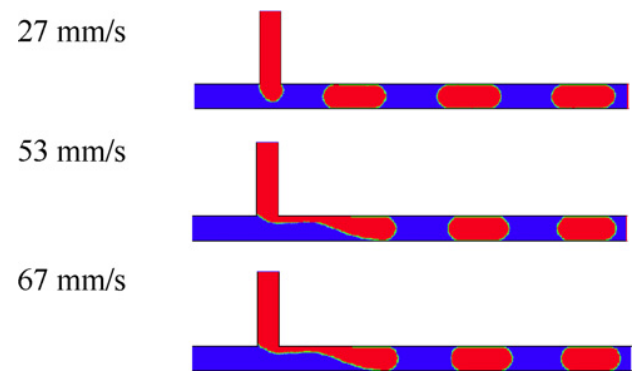

Fig. 8 - CFD simulated snapshots for the operating conditions corresponding to Fig. 7. 


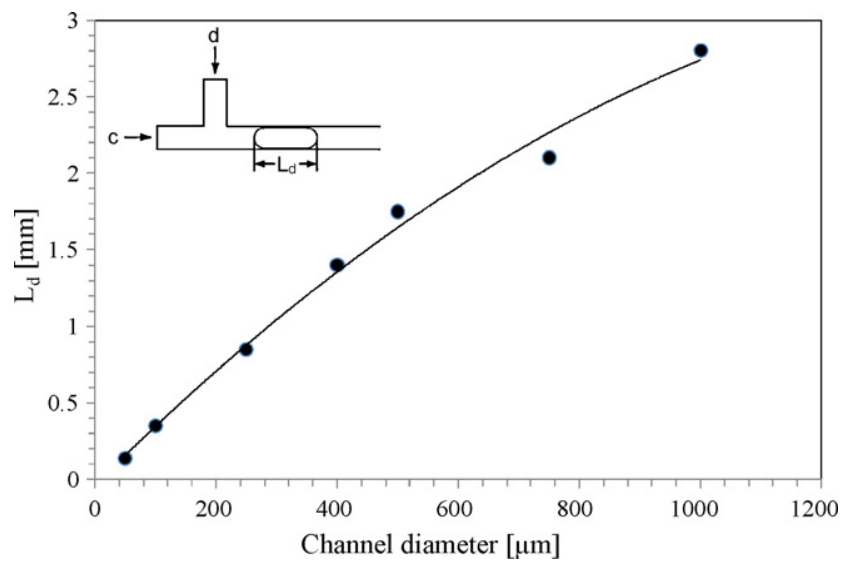

Fig. 9 - Length of the dispersed phase slug as a function of channel diameter $\left(U=27 \mathrm{~mm} / \mathrm{s}, \varphi_{d}=0.5\right)$.

\subsection{Scaling-up}

In order to study the effect of scaling-up on the slug size, simulations were carried out for different channel diameters ranging from $0.05 \mathrm{~mm}$ to $1 \mathrm{~mm}$ at low flow velocity where CFD simulations are in good agreement with the experimental results. The mesh size was about $1 \mu \mathrm{m}$ for channel diameter from $0.05 \mathrm{~mm}$ to $0.5 \mathrm{~mm}$ and was doubled for the simulations above $0.5 \mathrm{~mm}$ channel diameter. However, the $C_{r}$ (Eq. (6)) was fixed to 0.25 . The results for the dispersed phase slug length were plotted as a function of channel diameter for a similar flow velocity in Fig. 9. As it can be seen, for a channel below $0.5 \mathrm{~mm}$, it shows a linear relationship, however, with a further increase in the diameter, it shows only a slight decrease in slug length for a higher channel diameter.

\section{Conclusions}

A volume of fluid (VOF) based methodology was developed and simulations were carried out to study the slug flow generation for liquid-liquid systems. It was shown that the VOF method describes the slug flow generation and its key characteristics such as wall film and wetting behaviour very well. The obtained results show that slug flow generation can be characterised based on flow velocity $(U)$ and interfacial velocity $(\gamma / \mu)$ and is represented by Capillary number $(\mathrm{Ca}=U \mu / \gamma)$. To obtain a well-defined slug flow, the flow velocity should be much lower than the interfacial velocity-less than $1 \%$, which corresponds to a Ca of 0.01 . The simulations carried out for different channel dimensions show that the slug length can be preserved upto a channel diameter of $1 \mathrm{~mm}$. A study on the effect of different inlet geometries and operating conditions on the slug size is in progress.

\section{References}

Bico, J. and Quere, D., 2000, Liquid trains in a tube. Europhysics Letters, 51: 546-550.

Bothe, D., Koebe, M., Wielage, K. and Warnecke, H.-J., 2003, VOF simulations of mass transfer from single bubbles and bubble chains rising in the aqueous solutions, In FEDSM03: Fourth ASME-JSME Joint Fluids Engineering Conference Honolulu, Hawaii, USA.,

Brackbill, J.U., Kothe, D. and Zemach, C., 1992, A continuum method for modeling surface tension. Journal of Computational Physics, 100(2): 335-354.
Burns, J.R. and Ramshaw, C., 1999, Developement of a microreactor for chemical production. Transaction of the Institution of Chemical Engineers, 77A: 206-211.

Cerne, G., Petelin, S. and Tiselj, I., 2001, Coupling of the interface tracking and the two-fluid models for the simulation of incompressible two-phase flow. Journal of Computational Physics, 171(2): 776-804.

Chen, L. and Li, Y., 1998, A numerical method for two-phase flows with an interface. Environmental Modelling and Software, 13(3-4): 247-255.

Dessimoz, A.L., Cavin, L., Renken, A. and Kiwi-Minsker, L., 2008, Liquid-liquid two-phase flow patterns and mass transfer characteristics in rectangular glass microreactors. Chemical Engineering Science, 63(16): 4035-4044.

Dummann, G., Quittmann, U., Groschel, L., Agar, D.W., Worz, O. and Morgenschweis, K., 2003, The capillary-microreactor: a new reactor concept for the intensification of heat and mass transfer in liquid-liquid reactions. Catalysis Today, 79(1-4): 433-439.

(2006). FLUENT 6.3 user's guide. (Fluent Inc).

Garstecki, P., Fuerstman, M.J., Stone, H.A. and Whitesides, G.M., 2006, Erratum: formation of droplets and bubbles in a microfluidic T-junction-scaling and mechanism of break-up (Lab on a Chip-Miniaturisation for Chemistry and Biology (2006) 6 (437) DOI: 10.1039/b510841a). Lab on a Chip-Miniaturisation for Chemistry and Biology, 6(5): 693-694.

Garstecki, P., Fuerstman, M.J., Stone, H.A. and Whitesides, G.M., 2006, Formation of droplets and bubbles in a microfluidic T-junction-scaling and mechanism of break-up. Lab on a Chip-Miniaturisation for Chemistry and Biology, 6(3): 437-446.

Gupta, R., Fletcher, D.F. and Haynes, B.S., 2009, On the CFD modelling of Taylor flow in microchannels. Chemical Engineering Science, 64(12): 2941-2950.

Hirt, C.W. and Nichols, B.D., 1981, Volume of fluid (VOF) method for the dynamics of free boundaries. Journal of Computational Physics, 39(January (1)): 201-225.

Kashid MN. Experimental and modelling studies on liquid-liquid slug flow capillary microreactors. PhD, University of Dortmund; 2007.

Kashid, M.N., Platte, F., Agar, D.W. and Turek, S., 2007, Computational modelling of slug flow in a capillary microreactor. Journal of Computational and Applied Mathematics, 203(2 Spec. iss.): 487-497.

Kashid, M.N., Agar, D.W. and Turek, S., 2007, CFD modelling of mass transfer with and without chemical reaction in the liquid-liquid slug flow microreactor. Chemical Engineering Science, 62(18-20 SPEC. ISS.): 5102-5109.

Kumar, V., Vashisth, S., Hoarau, Y. and Nigam, K.D.P., 2007, Slug flow in curved microreactors: hydrodynamic study. Chemical Engineering Science, 62(24): 7494-7504.

Li, J. and Renardy, Y., 2000, Numerical study of flows of two immiscible liquids at low Reynolds number. SIAM Review, 42(3): 417-439.

Qian, D. and Lawal, A., 2006, Numerical study on gas and liquid slugs for Taylor flow in a T-junction microchannel. Chemical Engineering Science, 61(23): 7609-7625.

Tice, J.D., Song, H., Lyon, A.D. and Ismagilov, R.F., 2003, Formation of droplets and mixing in multiphase microfluidics at low values of the Reynolds and the capillary numbers. Langmuir, 19(22): 9127-9133.

Tice, J.D., Lyon, A.D. and Ismagilov, R.F., 2004, Effects of viscosity on droplet formation and mixing in microfluidic channels. Analytica Chimica Acta, 507(1): 73-77.

Van Sint Annaland, M., Dijkhuizen, W., Deen, N.G. and Kuipers, J.A.M., 2006, Numerical simulation of behavior of gas bubbles using a 3-D front-tracking method. AIChE Journal, 52(1): 99-110.

Zhao, Y., Chen, G. and Yuan, Q., 2007, Liquid-liquid two-phase mass transfer in the T-junction microchannels. AIChE Journal, 53(12): 3042-3053. 